Full metadata for this item is available in Research@StAndrews:FullText at:

http://research-repository.st-andrews.ac.uk/

\title{
Limitations to the cultural ratchet effect in young children
}

Tennie, C., Walter, V., Gampe, A., Carpenter, M. \& Tomasello, M.

\begin{tabular}{|l|l|}
\hline Date of deposit & 09032016 \\
\hline Version & This is an author version of this work. \\
\hline Access rights & $\begin{array}{l}\text { Copyright @ 2014 Elsevier Inc. All rights reserved. This work is } \\
\text { made available online in accordance with the publisher's policies. } \\
\text { This is the author created, accepted version manuscript following } \\
\text { peer review and may differ slightly from the final published } \\
\text { version. }\end{array}$ \\
\hline $\begin{array}{l}\text { Citation for } \\
\text { published version }\end{array}$ & $\begin{array}{l}\text { Tennie, C., Walter, V., Gampe, A., Carpenter, M., \& Tomasello, M. } \\
\text { (2014). Limitations to the cultural ratchet effect in young } \\
\text { children. Journal of Experimental Child Psychology, 126, 152-160. }\end{array}$ \\
\hline $\begin{array}{l}\text { Link to published } \\
\text { version }\end{array}$ & http://dx.doi.org/10.1016/j.jecp.2014.04.006 \\
\hline
\end{tabular}




\begin{abstract}
Although many animal species show at least some evidence of cultural transmission, broadly defined, only humans show clear evidence of cumulative culture. In the current study we investigated whether young children show the 'ratchet effect,' an important component of cumulative culture: the ability to accumulate efficient modifications across generations. We tested sixteen diffusion chains - altogether consisting of 80 children - to see how they solved an instrumental task (i.e., carrying something from one location to another). We found that when the chain was seeded with an inefficient way of solving the task, 4-year-old children were able to innovate and transmit these innovations so as to reach a more efficient solution. However, when it started out with relatively efficient solutions already (i.e., the ones the children in a control condition discovered for themselves), then there were no further techniques invented and/or transmitted beyond that. Thus young children showed the ratchet effect to a limited extent, accumulating efficient modifications but not going beyond the inventive level of the individual.
\end{abstract}

Keywords: ratchet effect, cultural evolution, imitation, social learning, diffusion chain, accumulation 


\section{Limitations to the cultural ratchet effect in young children}

Human culture, unlike that of other animal species, accumulates modifications over time (Dean, Vale, Laland, Flynn, \& Kendal, 2013; Tennie, Call, \& Tomasello, 2009; Tomasello, 1999). Humans socially learn from others, and once one individual makes an improvement in technique then others may adopt the improved method, so that later generations may eventually only have the improved version as an object of their social learning. This process has been dubbed the ratchet effect, as it typically leads to continual improvements over historical time (Tomasello, Kruger \& Ratner, 1993).

Recently, researchers have begun to study various types of cultural accumulation in the laboratory with both adults (e.g., Bangerter, 2000; Caldwell \& Millen, 2008, 2009; Kirby, Cornish, \& Smith, 2008; Mesoudi \& Whiten, 2004) and children (e.g., Flynn, 2008; Flynn \& Whiten, 2008, Hopper, Flynn, Wood, \& Whiten, 2010; Horner, Whiten, Flynn, \& de Waal, 2006). The basic technique is to create "generations" of learners in a diffusion chain (B learns from A, then C learns from B, then D learns from C, etc.). Using such a method, Caldwell and Millen have shown that improvements in simple construction tasks can accumulate across generations of adult learners. Flynn and Whiten (2008) found that 3- and 5-year-old children can transmit observed techniques across generations as well, and that the fidelity of transmission increases with age (see Flynn, 2008, for similar results with 2- and 3-year-olds, and see Horner et al., 2006, for a comparison of children to chimpanzees). In contrast, McGuigan and Graham (2009) found that 3-year-olds copied irrelevant actions in diffusion chains more robustly than did 5-year olds. Flynn (2008) also found a loss of irrelevant actions across diffusion chains in 2- and 3-year olds, and argued that such a loss of irrelevant actions can be interpreted as some form of cultural evolution, because the action sequence becomes more streamlined and efficient when irrelevant actions are eliminated (a "subtractive"

version of the ratchet effect). But none of these studies has asked whether children would also show what we will call the "additive ratchet effect," that is, inventing and accumulating improvements 
across generations in a manner similar to adults. This is what we did in the current study.

We tested children in diffusion chains in an experimental and a baseline condition. In the experimental condition an adult experimenter started each diffusion chain with a demonstration (presented pedagogically ${ }^{1}$ ). The experimenter demonstrated techniques that were not the most efficient given the materials available, in order to leave room for improvement. We compared children's performance in the experimental condition to that in the baseline condition in which no previous demonstration was given (the adult simply explained the goal of the task to children). Each diffusion chain consisted of five children, who were tested in succession (that is, only one child at a time observed either the experimenter or the preceding child). We used a task that was novel for the children: transporting dry rice from one location to another.

\section{Method}

\section{Participants}

Eighty 4-year-old children ( $M=4$ years, 5 months; range $=4 ; 3-4 ; 8 ; 40$ boys and 40 girls $)$ participated in the study. All were tested with a diffusion chain method in eight chains of five boys each $(\mathrm{N}=40)$ and eight chains of five girls each $(\mathrm{N}=40)$. We separated boys and girls because previous publications showed gender effects in copying in diffusion chains (Flynn \& Whiten, 2008). Data from an additional five children were excluded from the final analysis because of generation 1's (G1s') reluctance to perform demonstrations. Children were recruited and tested in their kindergartens, which were located in a mid-sized city in Germany. Their parents had given written permission for them to participate in child development studies.

\section{Materials}

We administered a novel rice transporting task, for which we used a transparent box $(58 \mathrm{x}$

\footnotetext{
${ }^{1}$ A pilot study, the results of which are available from the first author, revealed few effects of whether the demonstration was presented pedagogically or not, but order effects that were present in the analyses limited what we could conclude from it. Still, in it we replicated the finding of Flynn (2008) and McGuigan and Graham (2009) that irrelevant actions can be eliminated by chains of children.
} 
$39 \mathrm{x} 18 \mathrm{~cm}$ ) filled with approximately $12 \mathrm{~kg}$ of dry white rice. The task was to carry as much rice as possible from this box to a transparent bucket (diameter $=28 \mathrm{~cm}$, height $=30 \mathrm{~cm}$ ) located $2 \mathrm{~m}$ away. There was a scale under the bucket to weigh the amount of rice in the bucket at the end of each trip. Five different tools were laid out - always in standardized positions, as depicted in Figure 1 around the box for children to use, if they so wished, to transport the rice: a small white piece of cardboard, a small metallic scoop, a big green piece of cardboard, a small transparent bowl, and a blue bucket. The different tools were designed to vary in the efficiency with which they could be used to transport the rice, with the tool that would be used for the adult demonstration in the experimental condition being the least effective one. This was the case, as was confirmed by children's results in the pilot study: For that study we measured the mean effectiveness of each tool for all children who used each tool. The most efficient rice-carrying tool was the bowl, with a mean of $817.5 \mathrm{~g}$ of rice carried in a trip. This was followed by the bucket, with a mean of $439.7 \mathrm{~g}$, followed by the scoop with a mean of $63.9 \mathrm{~g}$, followed by the tool $\mathrm{E}$ used in the demonstration, the small piece of cardboard, with a mean of $21.5 \mathrm{~g}$. Off to one side, between the box of rice and the target bucket, at a distance of $1.40 \mathrm{~m}$, there were two child-sized chairs in which the observing children sat before and/or during demonstrations. Off to the other side, and in the vicinity of the target destination (bucket), was a chair in which E sat during children's participation in the task. 
Figure 1. Materials used in the rice task. The tool that was used by E is circled. [black-and-white in print]

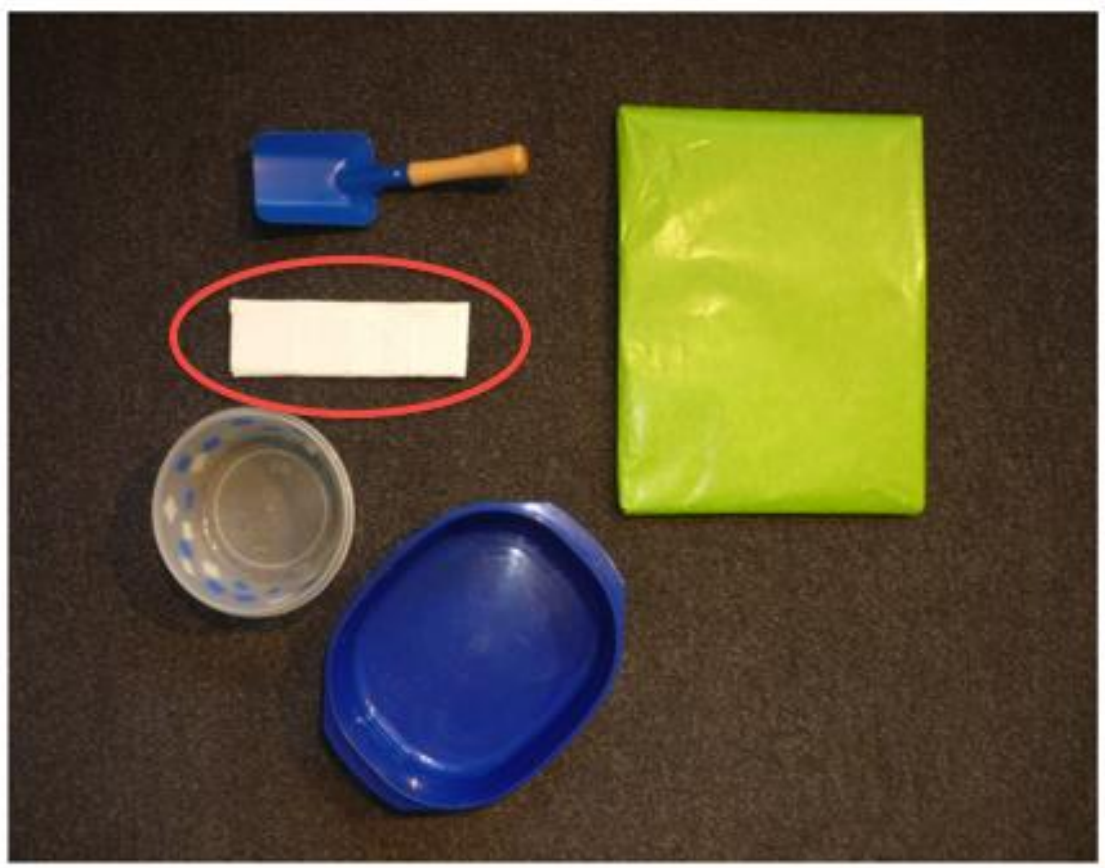

\section{Design}

Chains were randomly assigned to either the baseline condition or the experimental condition. Children in the baseline condition received no adult demonstration.

\section{Procedure}

Experimental condition. Children were tested in a quiet room in their kindergarten. Before testing, children first spent a few minutes in a different warm-up room, getting to know the experimenter (E) and the assistant and playing with the dry rice, in order to familiarize themselves with the rice. In the warm-up room they were told that they could have a turn in a special game. E and the assistant explained to children that before having their own turn in the upcoming game they would have to observe the child (or adult) before them. Then children (hereafter referred to as generations, from G1 to G5) drew lots to determine the order in which they would participate in the chain.

E and the first child (G1) then went to the testing room (the other children remained in the 
warm-up room playing with the assistant with unrelated toys). G1 sat on the floor facing $\mathrm{E}$ while $\mathrm{E}$ demonstrated the task: She bent down and scooped up some rice using the small white piece of cardboard as a tool and carried the rice over to the bucket and dumped it in. She performed this sequence of actions five times, which took a total of about $60 \mathrm{sec}$. During her demonstration, E talked about what she was doing in a normative and pedagogical way (e.g., "That's how we do it. We have to carry as much rice as we can to the bucket") and she looked ostensively at the child (for more procedural details, please see the Appendix).

During the demonstration phase, G1 observed. Then E replaced the tool she had used on the floor in its original position with the others and fetched G2 (while G1 waited on a chair in the testing room). G2 sat on one of the chairs and was instructed to observe G1 ("Watch, and then it is your turn"). E then said to G1, "Ok, [G1], now it is your turn with the rice! Take the rice over there!" G1 then was allowed 60 seconds to do the task while G2 watched. If G1 did not start, E repeated this instruction up to two times. If this was not successful the whole chain was then excluded ( $n=5)$. While G1 performed the demonstration, E sat next to the scale and made notes. She only gave neutral feedback to any questions concerning the task (e.g., "You can use what you like") and did not intervene in any discussions between G1 and G2. After his/her demonstration was over, G1 was taken back to the waiting room and G3 was brought to the testing room to serve as observer for G2's performance. The children in the waiting room were kept from talking about the task by the assistant after they had participated. This general procedure was repeated until all five generations had a chance to respond (for G5 there was no child observer). E was present, sitting near the bucket. Before she sat down there, for each generation she told the children that she had something else to do, after which she took out a notepad and pretended to write in it (in fact she was live-coding their responses).

Baseline condition. Children in the baseline condition experienced the same general procedure as children in the experimental condition, but they never received a demonstration from E (however, from G2 onwards, they received demonstrations from other children). Instead, in the 
warm-up room they were told that they could have a turn in a special game, and when they got to the testing room they were told that their task was to carry as much rice as possible to the bucket. Again, E was present, sitting near the bucket, and again she used the same notepad procedure as described above.

\section{Coding and Reliability}

Children's responses were coded from videotape separated by 'trips'. A trip was defined as starting when the child used a tool or their hand(s) to pick up some rice and as stopping when some rice was successfully carried to the goal destination. We recorded the duration (in seconds) of the trip and the type and number of tools used. E also recorded the amount of rice in the bucket (livecoded as measured by the scale) after each trip. From the coding of type and number of tools used, two other measures were calculated: the number of innovations (newly introduced tools) over E's demonstration (in the experimental condition) for each generation and the number of innovations over the previous generation for each generation (e.g., G3 over G2).

To assess inter-rater reliability, a random sample of $25 \%$ of the sessions was independently coded by a research assistant who was unaware of the aim of the study and which condition children were in. Reliability was perfect: $\mathrm{k}=1$ for type of tool used. The relation between copying $\mathrm{E}$ (or the previous generation) and innovating over $\mathrm{E}$ (or the previous generation) is complementary: a child either innovated or copied, and thus an increase in the number of children who copied is reflected by an equal decrease in the number of children who innovated. Reporting both cases would be redundant, and thus in the following we will report only our findings with regard to innovations. The research materials supporting this publication can be accessed by contacting the first author.

\section{Results}

Preliminary analyses revealed no effects of gender, age, or number of trips. Analyses were 
thus collapsed across these factors.

\section{Innovations}

A main question of interest was whether children copied E's and/or the previous child's choice of tool, or whether they innovated by introducing new tools - and whether they did this differently in the two conditions. Figure 2 presents an overview of the results concerning children's choice of tool in each generation in the experimental and baseline conditions.

Figure 2. Tool use in each of the five generations of each chain in each condition. (m: male child/chain, f: female child/chain). On the rare occasions that children used more than one tool in a single trip (this happened in two cases in the baseline condition and in one case in the experimental condition: f5's generation 1 used hand alone as well as scoop + bucket; m5's generation 5 used scoop + bowl as well as scoop + bucket; m2's generation 4 used cardboard as well as scoop), only the tool the children used most efficiently is depicted. Scoop + bucket and scoop + bowl mean that they were used in conjunction. 


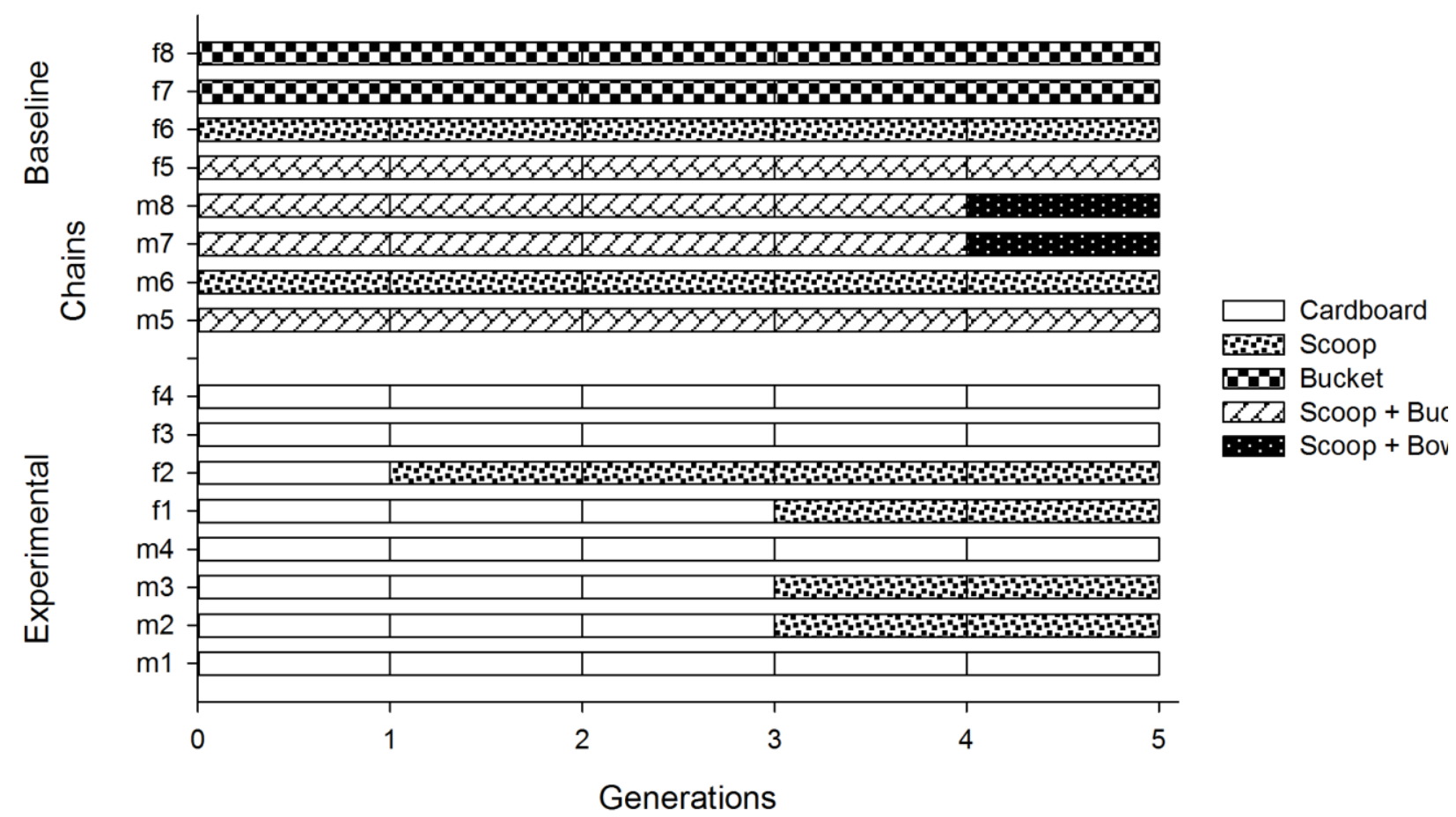

Choice of tool as innovation. For statistical reasons (to avoid problems with

pseudoreplication), we looked at innovations on the level of whole chains. On the chain level, we found a mean innovation rate over $\mathrm{E}$ (i.e., the mean number of generations in the chain who innovated over the demonstration E performed in the experimental condition) of 1.6 generations for the experimental condition and 5 generations for the baseline condition (for the baseline condition, these were "virtual innovations", since baseline children were not seeded by E's demonstration). This difference was significant (exact Mann-Whitney $U$ test, $U=4, n_{\text {experimental }}=8, n_{\text {baseline }}=8, p<$ 0.01). Children in the experimental condition thus innovated less than children in the baseline condition.

We also compared children's tendency to innovate over the tool the previous child generation used, for generations two to five (since G1s in the baseline control had no previous generation). This gives us a measure of children's innovations over peer demonstrations. We found a mean innovation rate over peers of 0.5 generations for the experimental condition and 0.375 generations for the baseline condition. This difference was not significant (exact Mann-Whitney $U$, 
$U=28, n_{\text {experimental }}=8, n_{\text {baseline }}=8, p=1$ ). Children thus innovated over their peers' demonstrations at similar rates in both conditions.

\section{Consequences for success}

Another main question of interest was whether the conditions had an effect on the success of the chains, and in particular whether children became more successful over the course of the diffusion chain - a hallmark of the ratchet effect. Figure 3 presents the success levels in the two conditions.

Figure 3. Mean amount of rice $(\mathrm{g})$ transported to the bucket in each condition for each generation (with standard deviations).

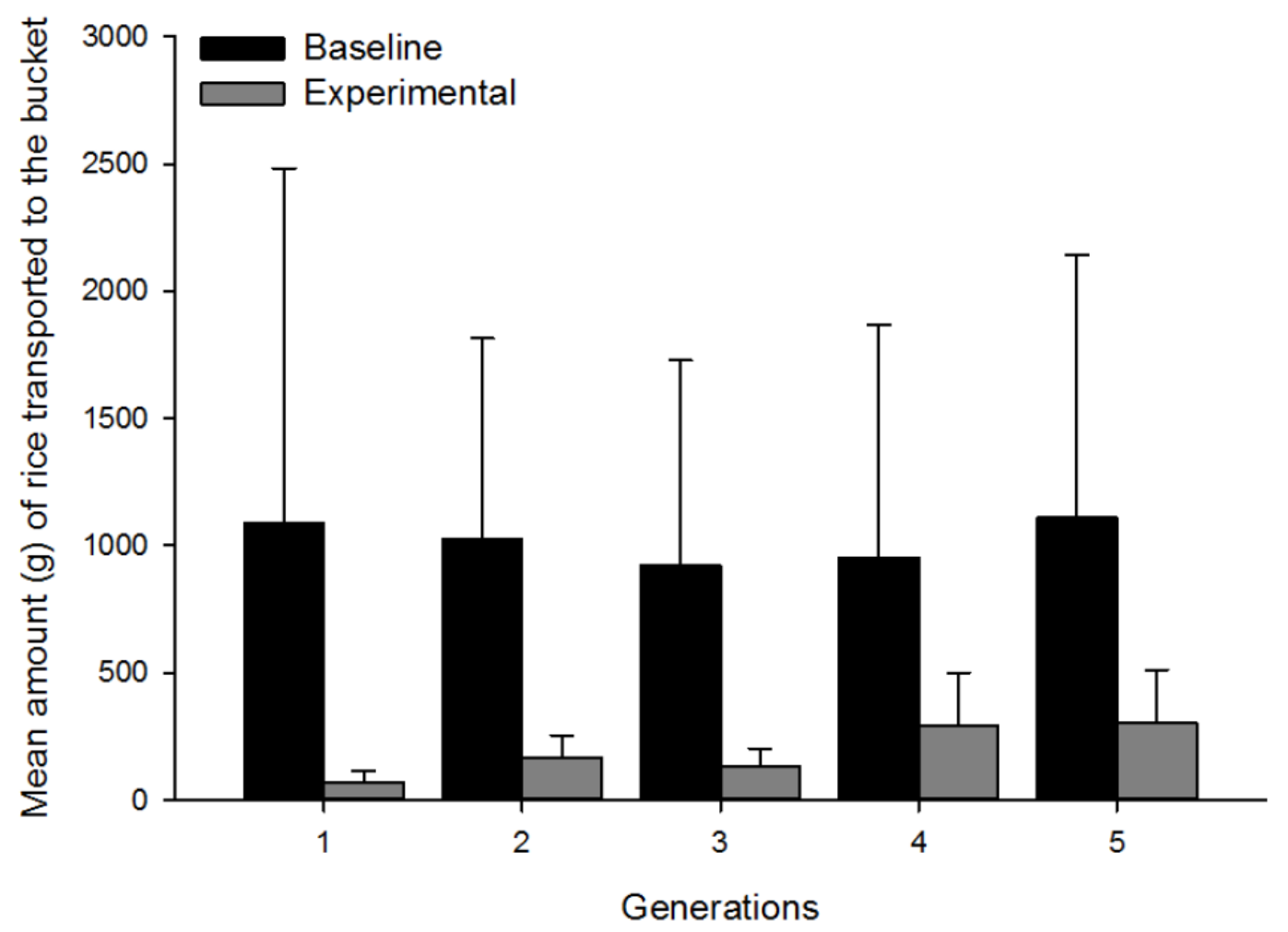

Overall success. Success rates differed in the two conditions: the chains were significantly more successful in the baseline condition $(M=5098 \mathrm{~g})$ than in the experimental condition $(M=962$ g) (exact Mann-Whitney $U$ test, $U=1, n_{\text {experimental }}=8, n_{\text {baseline }}=8, p<0.001$ ). Children who were seeded with E's inefficient demonstration thus were at a disadvantage to those who were not. 
Accumulation of success over time. We also checked for accumulation of success over the five generations. We analyzed accumulation of success by comparing each G1 with his/her respective G5 separately for each condition. In the experimental condition, G5s were found to be more successful $(M=304 \mathrm{~g})$ than G1s $\left(M=71 \mathrm{~g}\right.$; exact Mann-Whitney $U$ test, $U=8, n_{\mathrm{G} 1}=8, n_{\mathrm{G} 5}=$ $8, p=0.01)$. In the baseline condition, no difference between the success of G5s $(M=985 \mathrm{~g})$ and G1s $(M=1090 \mathrm{~g})$ was found (exact Mann-Whitney $U$ test, $\left.U=19, n_{\mathrm{G} 1}=8, n_{\mathrm{G} 5}=8, p=0.20\right)$. Thus, accumulation of success across generations was only found in the experimental, not in the baseline, condition.

Given that chains in the experimental condition did show accumulation of success, we next investigated at what level of success chains in the experimental condition ended up as compared with children in the baseline condition who had received no demonstration. We thus analyzed whether the chains in the experimental condition reached the same level of success in their G5s as did the G1s of the baseline chains. We found no difference between G5s of the experimental chains and G1s of the baseline chains (exact Mann-Whitney $U$ test, $U=21, n_{\mathrm{G} 1}=8, n_{\mathrm{G} 5}=8, p=0.27$ ). We corrected for multiple testing and the combined $p$ value reached significance (Fisher's Omnibus test, $\left.\chi^{2}(6, n=3)=15.12, p=0.02\right)$, indicating that these results were not simply a by-product of multiple testing. In summary, the success of baseline children (who were overall more successful than experimental children and who remained highly successful across generations) was only reached by experimental children at the end of the experimental chains (i.e., once they had accumulated success).

\section{Discussion}

The ratchet effect involves both copying and innovation: copying to provide a foundation on which to build and innovation for further improvements. Human cumulative culture is a product of both. In the current study we found a mix of copying and innovations. Overall, children copied some of the time, but they also sometimes introduced more efficient modifications. 
One of the main questions in this study was whether children would show improvement in efficiency across generations. We did find some improvement in efficiency, but only in a restricted sense. What we did not find was improvement in efficiency that went beyond the inventive level of the individual. Children in the baseline condition who had not seen adult demonstrations did not benefit over time from observing each other's solutions. These children transmitted efficient tool use across generations, and their lack of cultural evolution was due mainly to the fact that they detected the most efficient tools early on in the chain and so had few opportunities to improve their outcomes. However, given the constraints of the tools present, and the limited number of generations, it is possible that the chains could have produced cumulative culture, that is, behavior beyond the level of the individual, if given more time and opportunities. It was only the children who were seeded with inefficient demonstrations in the experimental condition who showed some form of improvement. However these children did not go beyond the inventive level of the individual either: By the end of their chains they were doing no better than children in the baseline condition. In our view what happened is that these children merely "recovered" their natural behavior across the chains, since eventually they reached success levels that were on a par with the success levels found in the baseline condition. Thus, while we have found evidence for some kind of ratchet effect in these children, we found it only in a specific sense: we found the ratchet extinguishing inefficient behavior, especially that which we had introduced experimentally.

Our finding that children extinguish inefficient behavior in diffusion chains is reminiscent of the finding that children extinguish unnecessary actions in diffusion chains (Flynn, 2008; McGuigan \& Graham, 2009). In an important sense, these studies, as well as our own, have therefore shown evidence for the subtractive ratchet effect in children, that is, one in which superfluous behaviors (Flynn, 2008; McGuigan \& Graham, 2009) or inefficient choices (this study) are eliminated.

What currently remains to be shown in children is an "additive" ratchet effect, where techniques are added or changed that lead, eventually, to efficient behavior outside the reach of the 
individual (e.g., Tennie, et al., 2009). It is possible that young children do not show the additive type of ratchet effect at all. Indeed, there is a growing literature on young children's tendency to "overimitate" or copy others "too" faithfully (e.g., Flynn, 2008; Lyons, Young, \& Keil, 2007; Nielsen \& Tomaselli, 2010), and this tendency might well hinder them from coming up with innovations. Indeed, in our rice carrying task, all first-generation children copied the adult's use of the most inefficient tool, the small piece of cardboard, when it was demonstrated pedagogically. It was only once children were observing peers, rather than adults, performing the task that the inefficient tool choices started to drop out - in line with other studies showing that children usually copy adults more closely than peers (for a review see Wood, Kendal, \& Flynn, 2013). Thus children's well-documented tendency to overimitate might compete with their tendency to innovate at this age.

However, given that this was the first study to test the presence of an additive ratchet effect, and given that our chains only consisted of a small number of generations, leaving little time for innovations to develop, it is too early to conclude that children do not show this effect. Both empirically and theoretically, this is a topic that is wide open in terms of future research. Empirically, future research on cumulative culture and related processes in children should include more chains, more generations within each chain, and more room for improvement. Furthermore, since faithful imitation is often related to the social context at the time of the demonstration and response (e.g., Nielsen \& Blank, 2011; Nielsen, Simcock, \& Jenkins, 2008; Over \& Carpenter, 2009, 2012; see also work on conformity and audience effects, e.g., Haun \& Tomasello, 2011), manipulating, for example, who is watching when could also be informative. It might also be interesting to manipulate the type of tool used in the demonstration. In the current study, none of the children in the baseline condition ever choose to use the small cardboard tool that was used by E during demonstrations in the experimental condition. Thus, this tool was probably regarded as very inefficient by the children (and/or perhaps was inconspicuous to them). Demonstrations using such a very "unlikely" tool might therefore have engendered additional pedagogical inferences in the 
children, which could have increased the copying fidelity for this type of tool (Csibra \& Gergely, 2006).

Along with future empirical work in this area, theoretically, more work needs to be done to distinguish different levels or types of cultural evolution, perhaps especially in children and in other animals who likely do not have fully developed skills of cumulative culture (for a start see Dean et al., 2013, and Tennie et al., 2009, who distinguish, for example, accumulations or 'step-wise traditions' from cumulative culture). Distinguishing between additive and subtractive ratchet effects, as we have done here, is one step in this direction. It will also help to consider different possible predictions for different situations. For example, one might expect very different results in instrumental contexts than in conventional or ritualistic or game contexts - in the latter, there should be very faithful copying and few or no innovations (Legare, Whitehouse, Herrmann, \& Wen, submitted; Nielsen, Cucchiaro, \& Mohamedally, 2012).

Given that cumulative culture is one of the key group-level processes that distinguishes humans from other animal species, it is important to explore all of its dimensions. Here we have shown that if an instrumental action is inefficient in a problem-solving context, even young children have the ability to eliminate it (by choosing a different tool) during the social learning process, leading to some improvements in problem-solving across generations. 


\section{References}

Bangerter, A. (2000). Transformation between scientific and social representations of conception: The method of serial reproduction. British Journal of Social Psychology, 39, 521-535.

Bonawitz, E., Shafto, P., Gweon, H., Goodman, N. D., Spelke, E., \& Schulz, L. (2011). The doubleedged sword of pedagogy: Instruction limits spontaneous exploration and discovery. Cognition, 120, 322-330.

Caldwell, C. A., \& Millen, A. E. (2008). Studying cumulative cultural evolution in the laboratory. Philosophical Transactions of the Royal Society of London B Biological Sciences, 363, 3529-3539.

Caldwell, C. A., \& Millen, A. E. (2009). Social learning mechanisms and cumulative cultural evolution: Is imitation necessary? Psychological Science, 20, 1478-1483.

Casler, K., \& Kelemen, D. (2005). Young children's rapid learning about artifacts. Developmental Science, 8, 472-480.

Csibra, G., \& Gergely, G. (2006). Social learning and social cognition: The case for pedagogy. In Y. Munakata \& M. H. Johnson (Eds.), Processes of change in brain and cognitive development. Attention and performance XXI (pp. 249 - 274). Oxford: Oxford University Press.

Csibra, G., \& Gergely, G. (2009). Natural pedagogy. Trends in Cognitive Sciences, 13, 148-153.

Dean, L. G., Vale, G. L., Laland, K. N., Flynn, E., \& Kendal, R. L. (2013). Human cumulative culture: A comparative perspective. Biological Reviews. Online First.

Flynn, E. (2008). Investigating children as cultural magnets: Do young children transmit redundant information along diffusion chains? Philosophical Transactions of the Royal Society of London B Biological Sciences, 363, 3541-3551.

Flynn, E., \& Whiten, A. (2008). Cultural transmission of tool use in young children: A diffusion chain study. Social Development, 17, 699-718. 
Haun, D. B. M., \& Tomasello, M. (2011). Conformity to peer pressure in preschool children. Child Development, 82, 1759-1767.

Hopper, L. M., Flynn, E. G., Wood, L. A. N., \& Whiten, A. (2010). Observational learning of tool use in children: Investigating cultural spread through diffusion chains and learning mechanisms through ghost displays. Journal of Experimental Child Psychology, 106, 82-97.

Horner, V., Whiten, A., Flynn, E., \& de Waal, F. B. M. (2006). Faithful replication of foraging techniques along cultural transmission chains by chimpanzees and children. Proceedings of the National Academy of Sciences of the United States of America, 103, 13878-13883.

Kirby, S., Cornish, H., \& Smith, K. (2008). Cumulative cultural evolution in the laboratory: An experimental approach to the origins of structure in human language. Proceedings of the National Academy of Sciences of the United States of America, 105, 10681-10686.

Legare, C. H., Whitehouse, H., Herrmann, P., \& Wen, N. (submitted). Imitative foundations of cultural learning.

Lyons, D.E., Young, A.G., \& Keil, F.C. (2007). The hidden structure of overimitation. Proceedings of the National Academy of Sciences, 104, 19751-19756.

McGuigan, N., \& Graham, M. (2009). Cultural transmission of irrelevant tool actions in diffusion chains of 3- and 5-year-old children. European Journal of Developmental Psychology, 7, $561-577$.

Mesoudi, A., \& Whiten, A. (2004). The hierarchical transformation of event knowledge in human cultural transmission. Journal of Cognition and Culture, 4, 1-24.

Nielsen, M., \& Blank, C. (2011). Imitation in young children: When who gets copied is more important than what gets copied. Developmental Psychology, 47 (4), 1050-1053.

Nielsen, M., Cucchiaro, J., \& Mohamedally, J. (2012). When the transmission of culture is child's play. PLoS ONE, 7, e34066.

Nielsen, M., Simcock, G., \& Jenkins, L. (2008). The effect of social engagement on 24-montholds' imitation from live and televised models. Developmental Science, 11 (5), 722-731. 
Nielsen, M., \& Tomaselli, K. (2010). Overimitation in Kalahari Bushman children and the origins of human cultural cognition. Psychological Science, 21 (5), 729-736.

Over, H., \& Carpenter, M. (2009). Priming third-party ostracism increases affiliative imitation in children. Developmental Science, 12 (3), F1-F8.

Over, H., \& Carpenter, M. (2012). Putting the social into social learning: Explaining both selectivity and fidelity in children's copying behavior. Journal of Comparative Psychology, $126(2), 182-192$.

Schmidt, M. F. H., Rakoczy, H., \& Tomasello, M. (2011). Young children attribute normativity to novel actions without pedagogy or normative language. Developmental Science, 14, 530539.

Tennie, C., Call, J., \& Tomasello, M. (2009). Ratcheting up the ratchet: On the evolution of cumulative culture. Philosophical Transactions of the Royal Society of London B Biological Sciences, 364, 2405-2415.

Tomasello, M. (1999). The cultural origins of human cognition. Cambridge, MA: Harvard University Press.

Tomasello, M., Kruger, A. C., \& Ratner, H. H. (1993). Cultural learning. Behavioral and Brain Sciences, 16, 495-511.

Wood, L. A., Kendal, R. L., \& Flynn, E. G. (2013). Whom do children copy? Model-based biases in social learning. Developmental Review, 33 (4), 341-356. 


\title{
Appendix
}

What E said to children in each condition.

\section{Experimental Condition}

\section{Baseline Condition}

E faces child and squats down, so that eye contact is established.

E to G1: "Let me explain this to you. One has to E to G1 "Let me explain this to you. You have bring as much rice as one can to the bucket!” to bring as much rice as you can to the bucket!”

\section{"Do you see all of these things?"}

E points to the tools.

\begin{abstract}
"We could use these to carry as much rice as we "You could use these to carry as much rice as possibly can to the bucket." you possibly can to the bucket."
\end{abstract}

"But before it is your turn, I will show you how

we do it, ok? And afterwards it is your turn!

Stay seated there, this is how we do it."

E stands up and takes the (inefficient) tool, says to G1:

“Look!”

E then starts transporting rice.

\footnotetext{
After the second demonstration E says to G1:

"This is how we do it."

And after the fifth demonstration E says to G1:
"This is how we do it. I'll go and get G2. Then it "I'll go and get G2. Then it is your turn." is your turn."

